\title{
Cyclic Cushing's disease with misleading inferior petrosal sinus sampling results during a trough phase
}

\author{
Vivien Bonert, MD, ${ }^{1}$ Namrata Bose, MD, ${ }^{2}$ and John D. Carmichael, MD² \\ ${ }^{1}$ Pituitary Center, Cedars-Sinai Medical Center; and ${ }^{2}$ USC Pituitary Center, Keck School of Medicine of University of Southern \\ California, Los Angeles, California
}

Diagnosing Cushing's syndrome is challenging and is further hampered when investigations are performed in a patient with cyclic Cushing's syndrome. A subset of patients with Cushing's syndrome exhibit periods of abnormal cortisol secretion with interspersed normal secretion. Patients can have periods of clinical improvement during these quiescent phases or remain symptomatic. Initial diagnostic testing can be challenging because of the unpredictable durations of the peak and trough phases, and it is especially challenging when the diagnosis of cyclic Cushing's syndrome has not yet been determined. Here, the authors present the case of a patient with Cushing's disease with a pathology-proven adrenocorticotropic hormone (ACTH)-secreting pituitary adenoma and whose initial inferior petrosal sinus sampling (IPSS) results were deemed indeterminate; further studies elucidated the diagnosis of cyclic Cushing's syndrome. Repeat IPSS was diagnostic of a central source for ACTH secretion, and the patient was treated successfully with transsphenoidal resection. Literature concerning the diagnosis and management of cyclic Cushing's syndrome is also reviewed.

http://thejns.org/doi/abs/10.3171/2014.12.FOCUS14780

KEY WORDS cyclic Cushing's disease; diagnosis; pitfalls

$\mathrm{D}$ IAGNOSING Cushing's syndrome (CS) is challenging, and there are several potential pitfalls. A subset of patients exhibit cyclic or periodic hypersecretion of cortisol with intermittent periods of normal secretion. A variety of presentations exist, including regular cyclic hormone secretion with or without variations in clinical symptoms and irregular cycles of cortisol secretion with or without corresponding changes in phenotypic expression..$^{10}$ Periods of normalcy can last weeks, months, or years between cycles, with abnormal periods also varying significantly. ${ }^{1}$ Making the correct diagnosis can be hampered by these normal periods, and results of testing can lead to errors in the differential diagnosis. Here, we present an interesting case of cyclic CS in a patient who was ultimately found to have Cushing's disease from an adrenocorticotropic hormone (ACTH)-producing pituitary adenoma.

\section{Case Report}

A 63-year-old woman with a medical history of resistant hypertension presented with complaints of intermittent weight gain, bruising, progressive fatigue, and emotional lability. On examination, she was noted to have classic cushingoid features with moon facies, plethora, central obesity, upper arm and leg muscle atrophy, and bruises. Her blood pressure was 110/74 mm Hg, her weight was $180 \mathrm{lbs}$, her height was 61 inches, and her body mass index was 34 $\mathrm{kg} / \mathrm{m}^{2}$. The neurological examination revealed that she had an inability to rise from a squatting position.

Investigations

Biochemistry. Two 24-hour urinary free cortisol (UFC) tests were completed and revealed elevated levels of 225 and $175 \mu \mathrm{g} / 24$ hours (reference range $<50 \mu \mathrm{g} / 24$

ABBREVIATIONS ACTH = adrenocorticotropic hormone; $\mathrm{CRH}=$ corticotropin-releasing hormone; CS = Cushing's syndrome; DDAVP = desmopressin acetate; IPSS = inferior petrosal sinus sampling; UFC = urinary free cortisol.

SUBMITTED November 19, 2014. ACCEPTED December 8, 2014.

INCLUDE WHEN CITING DOI: 10.3171/2014.12.FOCUS14780.

DISCLOSURE The authors report no conflict of interest concerning the materials or methods used in this study or the findings specified in this paper. 
hours). Her plasma ACTH level was $26 \mathrm{pg} / \mathrm{ml}$ (reference range 5-27 pg/ml). A 1-mg overnight dexamethasone suppression test revealed a morning serum cortisol concentration of $22 \mu \mathrm{g} / \mathrm{dl}$ (reference range $<1.8 \mu \mathrm{g} / \mathrm{dl}$ ), and a low-dose 2-day (2 mg/day) dexamethasone suppression test revealed 24-hour UFC levels that were suppressed from 48 to $38 \mu \mathrm{g} / 24$ hours (reference range $>50 \%$ suppression). A high-dose (8-mg) overnight dexamethasone suppression test revealed a pretreatment serum cortisol level of $21 \mu \mathrm{g} / \mathrm{dl}$, which was suppressed to $5.4 \mu \mathrm{g} / \mathrm{dl}$ after dexamethasone administration. Her insulin-like growth factor-1 level was $76 \mathrm{ng} / \mathrm{ml}$, her prolactin level was 8.3 $\mathrm{ng} / \mathrm{ml}$, her free thyroxine level was $1.3 \mathrm{ng} / \mathrm{dl}$, her thyroidstimulating hormone level was $0.7 \mathrm{mIU} / \mathrm{L}$, her luteinizing hormone level was $<0.2 \mathrm{IU}$, and her follicle-stimulating hormone level was < 0.7 IU (inappropriately suppressed for a postmenopausal woman). The results of an oral glucose-tolerance test were consistent with impaired glucose tolerance.

Imaging. Pituitary MRI was suggestive of a small central hypointense lesion. A dual-energy x-ray absorptiometry scan confirmed osteoporosis.

\section{Follow-Up}

With a diagnosis of ACTH-dependent CS, the patient underwent the initial inferior petrosal sinus sampling (IPSS) (Table 1), 7 months after her presentation and initial workup from her referring endocrinologist. The IPSS results revealed a $<2: 1$ central-to-peripheral gradient at baseline, with a $<3: 1$ gradient of central-to-peripheral ACTH in the petrosal sinus samples after corticotropinreleasing hormone (CRH) administration. An isolated central-to-peripheral gradient was seen $(>3: 1)$ in samples obtained from the jugular vein, but it was believed to be inconsistent with petrosal samples and nondiagnostic. Without a clear central-to-peripheral gradient between the petrosal samples and the peripheral measurements, the IPSS results were considered indeterminate and not supportive of a diagnosis of central ACTH-dependent Cushing's disease.

No additional samples for 24-hour UFC testing had been collected since the time of the patient's initial presentation. At that time, she reported that her symptoms associated with CS had resolved in the weeks leading up to the IPSS procedure. Her facial plethora and weakness had improved dramatically. It was decided to reevaluate the patient with further testing before proceeding with further investigation of the source of the ACTH. Serial samples for 24-hour UFC measurements (Fig. 1) were then collected for 11 months to document cortisol hypersecretion. A second IPSS procedure was scheduled during a cortisol hypersecretory phase (Table 2). The second IPSS procedure was diagnostic of a central source for ACTH, which supported a diagnosis of Cushing's disease. The peaks and troughs revealed by serial measurements of 24-hour UFC levels suggested a diagnosis of cyclic Cushing's disease.

\section{Treatment and Posttreatment Course}

The patient underwent transsphenoidal surgery, with removal of a microadenoma and the inferior central portion of the pituitary gland. Pathology confirmed a sparsely
TABLE 1. Initial IPSS

\begin{tabular}{|c|c|c|c|}
\hline \multirow[b]{2}{*}{$\begin{array}{l}\text { Specimen } \\
\text { Time (mins) }\end{array}$} & \multicolumn{2}{|c|}{ Petrosal ACTH (pg/ml) } & \multirow[b]{2}{*}{$\begin{array}{c}\text { Peripheral } \\
\text { ACTH }(p g / m l)\end{array}$} \\
\hline & $\mathrm{Lt}$ & Rt & \\
\hline-5 & 28 & 28 & 30 \\
\hline 0 & 27 & 28 & 29 \\
\hline 3 & 28 & 28 & 27 \\
\hline 5 & 75 & 91 & 46 \\
\hline 10 & 123 & 125 & 93 \\
\hline \multicolumn{4}{|c|}{ Jugular Vein (pg/ml) } \\
\hline & Lt & $\mathrm{Rt}$ & \\
\hline 15 & 485 & 118 & 101 \\
\hline \multicolumn{4}{|c|}{ Superior Vena Cava (pg/ml) } \\
\hline 17 & & & 97 \\
\hline \multicolumn{4}{|c|}{ Inferior Vena Cava Supra-adrenal (pg/ml) } \\
\hline 19 & & & 99 \\
\hline \multicolumn{4}{|c|}{ Inferior Vena Cava Infra-adrenal (pg/ml) } \\
\hline 20 & & & 115 \\
\hline
\end{tabular}

granulated corticotroph adenoma $(\mathrm{Ki} 67<1 \%)$ that was p53 negative. Postoperatively, her serum cortisol concentration reached a nadir of $1.1 \mu \mathrm{g} / \mathrm{dl}$ on Postoperative Day 2, falling from $9.6 \mu \mathrm{g} / \mathrm{dl}$ on Postoperative Day 1. She was discharged from the hospital after being placed on a hydrocortisone replacement regimen and reported symptoms of steroid withdrawal in the perioperative time period. She lost $8 \mathrm{lbs}$, complained of joint aches, and experienced resolution of her hypertension without taking her antihypertensive medication. Her recovery was otherwise uneventful, and she returned to her referring endocrinologist for long-term follow-up.

\section{Discussion}

This case clearly documents several peaks and troughs of cortisol secretion as measured by frequent 24-hour sample collections for UFC testing. The results of IPSS performed during a trough phase were more consistent with a peripheral source of ACTH, given the lack of gradient between the petrosal and peripheral samples at baseline and after $\mathrm{CRH}$ administration, but were indeterminate at the time and required further investigation. Although it was not recognized at the time, one potential explanation for the results was that the patient had cyclic CS and was studied during a trough phase. Another potential source for error was improper cannulation of the petrosal sinuses leading to the errant measurement of ACTH levels. Both of these situations necessitate repeat performance of IPSS. The correct diagnosis was made by restudying the patient after demonstrating periodicity of cortisol secretion and ensuring that the IPSS was performed during a phase of active cortisol secretion.

CS is an important clinical entity with several deleterious effects on health, including dyslipidemia, impaired glucose tolerance, hypertension, bone loss, proximal muscle wasting, weight gain, and mood disturbances. In classic CS, cortisol levels are elevated without diurnal varia- 


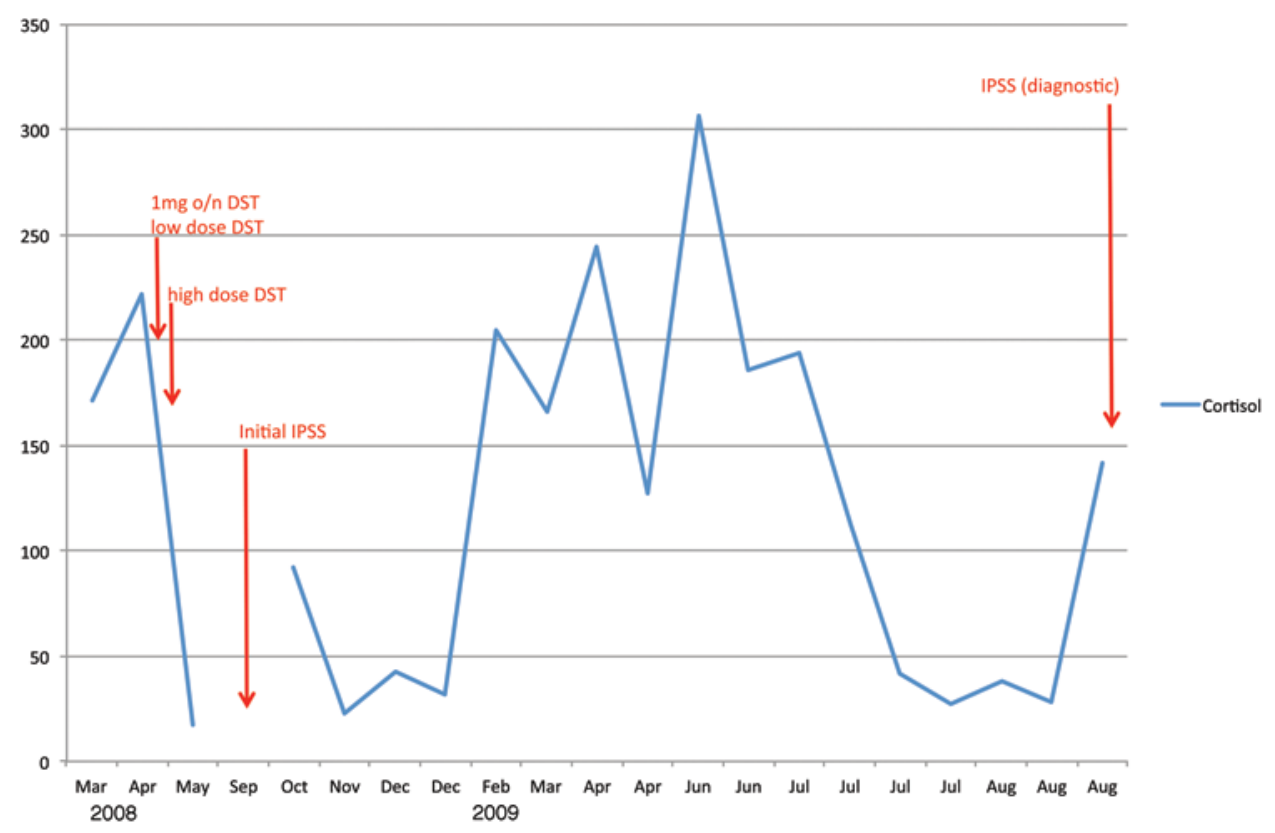

FIG. 1. Laboratory evaluation of serial measurements of 24-hour UFC levels revealed 3 distinct peaks and 2 trough periods. Collections for 24-hour UFC were often obtained multiple times per month. Dexamethasone suppression testing and IPSS are indicated by the arrows. o/n DST = overnight dexamethasone suppression test.

tion, and normal feedback inhibition of cortisol secretion is diminished.

Cyclic CS is a rare subtype of CS in which periods of biochemical and/or clinical hypercortisolism alternate with periods of eucortisolism. A variety of subcategories have been described..$^{10}$ Cyclic patterns of biochemical hypercortisolism may be regular or irregular and have variable or constant clinical manifestations. Cycle durations have also been noted to vary between weeks and years. ${ }^{8}$

Although it is rarely diagnosed, cyclic CS may in fact be more frequent than commonly thought. Indeed, a retrospective study of 201 patients with CS found cyclic variability in $30(15 \%)$ of them. ${ }^{1}$ Difficulties in diagnosis

TABLE 2. Diagnostic IPSS

\begin{tabular}{|c|c|c|c|}
\hline \multirow[b]{2}{*}{$\begin{array}{l}\text { Specimen } \\
\text { Time (mins) }\end{array}$} & \multicolumn{2}{|c|}{ Petrosal ACTH (pg/ml) } & \multirow[b]{2}{*}{$\begin{array}{c}\text { Peripheral } \\
\text { ACTH (pg/ml) }\end{array}$} \\
\hline & $\mathrm{Lt}$ & Rt & \\
\hline-5 & 34 & 34 & 32 \\
\hline 0 & 31 & 30 & 27 \\
\hline 3 & 515 & 652 & 27 \\
\hline 5 & 579 & 1395 & 78 \\
\hline 10 & 233 & 243 & 216 \\
\hline \multicolumn{4}{|c|}{ Jugular Vein (pg/ml) } \\
\hline & Lt & $\mathrm{Rt}$ & \\
\hline 15 & 504 & 243 & 166 \\
\hline \multicolumn{4}{|c|}{ Superior Vena Cava (pg/ml) } \\
\hline 17 & & & 196 \\
\hline \multicolumn{4}{|c|}{ Inferior Vena Cava Supra-adrenal (pg/ml) } \\
\hline 19 & & & 181 \\
\hline \multicolumn{4}{|c|}{ Inferior Vena Cava Infra-adrenal (pg/ml) } \\
\hline 20 & & & 143 \\
\hline
\end{tabular}

underlie the underreporting of this phenomenon. As demonstrated in our case report, capturing a period of cortisol excess and confirming the diagnosis during this time period may be challenging. A generally accepted diagnostic criterion for cyclic CS is the recognition of 2 cycles (3 peaks and 2 troughs) of cortisol production, ${ }^{3}$ although others consider 1 complete cycle ( 2 peaks and 1 trough) sufficient for making the diagnosis. ${ }^{1}$

Any etiology of CS can produce cyclic patterns of hypercortisolism. In a review of 65 published cases of cyclic CS, $54 \%$ were caused by ACTH-producing pituitary adenomas, $26 \%$ were caused by ectopic ACTH production, and $11 \%$ were a result of ACTH-independent causes. ${ }^{8}$ The pathophysiology has yet to be defined clearly, although some theories have been proposed. A histological finding of necrosis within tumors led some investigators to propose that episodic hemorrhage and necrosis of cortisol-producing cells causes a cyclical release of cortisol, ${ }^{7}$ although others have identified cases of cyclical CS without necrosis in the tumor specimen. ${ }^{2}$ Another theory postulates that tumor calcifications lead to infarction with subsequent eucortisolemia; ${ }^{4}$ however, this theory does not explain the periodicity. A third theory involves potential hypothalamic causes as mediators of pulsatile ACTH hypersecretion. Neurotransmitters thought to be involved include $\mathrm{CRH}$, noradrenaline, dopamine, acetylcholine, and $\gamma$-aminobutyric acid. ${ }^{8}$

Diagnosis of typical CS is known to be challenging because no single test has ideal diagnostic accuracy, which results in the need for multiple tests to confirm a diagnosis. According to Endocrine Society practice guidelines, screening tests include 24-hour UFC measurement, midnight salivary cortisol measurement, and the 1-mg dexamethasone suppression test, with a note that urinary and salivary levels should be measured at least twice given the 
variability in results. ${ }^{9}$ A diagnosis of cyclical CS is complicated further by the relapse and remission of biochemical and clinical findings and the need for close monitoring and coordination of confirmatory tests. Furthermore, an increased duration of testing is required given the varying periods of remission, which are known to last up to 5 years. This poses a major obstacle in the ability to administer appropriate management in a timely fashion.

Novel diagnostic methods are being explored to circumvent this pitfall. Hair cortisol levels are known to be significantly elevated in patients with CS over those in the general population (sensitivity $86 \%$, specificity $98 \%$ ). ${ }^{6}$ The advantage of using hair cortisol levels is that they can provide data over the previous several months in one analytical setting. In one study, hair samples were obtained from one lock of hair each from healthy controls, patients with $\mathrm{CS}$, and patients with cyclic CS. ${ }^{6}$ Cortisol measurements at $1-\mathrm{cm}$ intervals correspond to monthly time frames. In patients with cyclic CS, hair cortisol-level fluctuations were found to correlate with the clinical course of CS. ${ }^{6}$ In another report, a desmopressin acetate (DDAVP) stimulation test was used for a case in which biochemical evidence was not confirmed, resulting in a 2-year delay of the eventual discovery of an ACTH-producing pituitary adenoma. ${ }^{5}$ Thus, both the analysis of hair cortisol and the DDAVP stimulation test may improve on the diagnostic approach by significantly shortening the time to diagnosis and enabling more timely management.

As with classic CS, the treatment of cyclical CS generally involves surgical removal of the tumor or, for patients who are not candidates for surgery, medical management. However, posttreatment management poses a unique set of challenges in these patients. Patients with cyclic CS caused by pituitary disease have a significantly higher recurrence rate postoperatively $(63 \%)$ and lower cure rates $(25 \%)$ than those of patients with noncyclical CS (approximately $80 \%$ cure rate) ${ }^{8}$ Moreover, postoperative management is complicated by the fact that normal cortisol levels in these patients may not be indicative of cure but instead correspond to a period of quiescence. In this situation, there is a need to closely monitor for longer periods of time, and perhaps more frequently than usual, to ensure the capture of a relapse if it occurs. It was fortunate that, in our case, the postoperative symptoms of cortisol withdrawal and the need for steroid replacement were strongly indicative of remission in this patient.

We report here a case that clearly documents multiple peaks and troughs of cortisol secretion, as measured by frequent sampling of 24-hour UFC levels. The IPSS performed during a trough phase provided misleading results because of the lack of gradient at baseline and after CRH administration. The correct diagnosis was made by repeating the IPSS and ensuring that it was performed during an active phase of cortisol secretion. It is important to recognize cyclic CS as a clinical entity and to note that its prevalence may be higher than currently believed given the challenges in establishing a diagnosis. A diagnosis of cyclic CS is made by showing peaks and troughs of cortisol secretion either by frequent measurement of cortisol levels in urine or saliva or perhaps by using novel techniques such as the analysis of hair cortisol levels or DDAVP stimulation testing. As illustrated by this case, the timing of IPSS (i.e., during a period of quiescence) may lead to an incorrect diagnosis and further delay appropriate management. It is important that testing for the differential diagnosis be done only during periods of biochemical or clinical hypercortisolism. Management of these patients is complicated by high recurrence rates and low cure rates, which necessitate the need for continued closer monitoring for longer durations of time than for patients with noncyclic CS.

\section{References}

1. Alexandraki KI, Kaltsas GA, Isidori AM, Akker SA, Drake WM, Chew SL, et al: The prevalence and characteristic features of cyclicity and variability in Cushing's disease. Eur J Endocrinol 160:1011-1018, 2009

2. Bailey RE: Periodic hormonogenesis-a new phenomenon. Periodicity in function of a hormone-producing tumor in man. J Clin Endocrinol Metab 32:317-327, 1971

3. Brown RD, Van Loon GR, Orth DN, Liddle GW: Cushing's disease with periodic hormonogenesis: one explanation for paradoxical response to dexamethasone. J Clin Endocrinol Metab 36:445-451, 1973

4. La Civita KA, McDonald S, Jacobson J: Cyclic Cushing's disease in association with a pituitary stone. South Med J 82:1174-1176, 1989

5. Leal-Cerro A, Martín-Rodríguez JF, Ibáñez-Costa A, Madrazo-Atutxa A, Venegas-Moreno E, León-Justel A, et al: Desmopressin test in the diagnosis and follow-up of cyclical Cushing's disease. Endocrinol Nutr 61:69-76, 2014

6. Manenschijn L, Koper JW, van den Akker EL, de Heide LJ, Geerdink EA, de Jong FH, et al: A novel tool in the diagnosis and follow-up of (cyclic) Cushing's syndrome: measurement of long-term cortisol in scalp hair. J Clin Endocrinol Metab 97:E1836-E1843, 2012

7. Mantero F, Scaroni CM, Albiger NM: Cyclic Cushing's syndrome: an overview. Pituitary 7:203-207, 2004

8. Meinardi JR, Wolffenbuttel BH, Dullaart RP: Cyclic Cushing's syndrome: a clinical challenge. Eur J Endocrinol 157:245-254, 2007

9. Nieman LK, Biller BM, Findling JW, Newell-Price J, Savage MO, Stewart PM, et al: The diagnosis of Cushing's syndrome: an Endocrine Society Clinical Practice Guideline. J Clin Endocrinol Metab 93:1526-1540, 2008

10. Shapiro MS, Shenkman L: Variable hormonogenesis in Cushing's syndrome. Q J Med 79:351-363, 1991

\section{Author Contributions}

Conception and design: Carmichael. Acquisition of data: Carmichael, Bonert. Analysis and interpretation of data: Carmichael, Bonert. Drafting the article: all authors. Critically revising the article: all authors. Reviewed submitted version of manuscript: all authors. Approved the final version of the manuscript on behalf of all authors: Carmichael.

\section{Correspondence}

John D. Carmichael, USC Pituitary Center, 1520 San Pablo Dr., No.3800, Los Angeles, CA 90033. email: john.carmichael@ med.usc.edu. 